\title{
Sequential rotation to insert a left double-lumen endotracheal tube using the GlideScope $\$$
}

\author{
Sergio Bustamante, MD • Iván Parra-Sánchez, MD • \\ John Apostolakis, MD
}

Received: 12 November 2009/Accepted: 30 November 2009/Published online: 9 January 2010

(C) Canadian Anesthesiologists' Society 2009

\section{To the Editor:}

The GlideScope ${ }^{\circledR}$ videolaryngoscope (GVL) (Verathon, Bothell, WA, USA) allows the glottic opening to be visualized easily. However, it poses difficulties when double-lumen endotracheal tubes (DLT) are advanced into the trachea. ${ }^{1,2}$ This suggests that the traditional method of advancing the DLT may be insufficient if used with the GVL. A combination of several factors can decrease the operator's ability to manipulate this tube proficiently, including the complex shape of the DLT, ${ }^{3}$ the angulation of the GVL blade, and the indirect viewing of the airway. ${ }^{4}$

A main feature of the DLT is the presence of a concave distal and a concave proximal curvature. Under direct laryngoscopy, the conventional method of insertion entails advancing the tube with the distal curvature directed anteriorly. Once the bronchial tip passes the vocal cords, the DLT is rotated $90^{\circ}$ counter clockwise, a rotation that facilitates the alignment of the bronchial tip with the left main bronchus. ${ }^{3}$

With the GVL, advancement of a single-lumen tube through the glottis may be difficult or impossible, because it tends to advance anteriorly and thus nearly perpendicular to the axis of the trachea. ${ }^{5}$ This difficulty can also arise during an attempt to advance a DLT with the GVL, because the distal concave curvature directs the tip of the bronchial lumen more anteriorly.

Electronic supplementary material The online version of this article (doi:10.1007/s12630-009-9243-2) contains supplementary material, which is available to authorized users.

S. Bustamante, MD (ه) · I. Parra-Sánchez, MD .

J. Apostolakis, MD

Anesthesiology Institute, Cleveland Clinic, Cleveland, USA

e-mail: bustams@ccf.org
Prior to its insertion, we pre-curve the distal $10-12 \mathrm{~cm}$ of the DLT approximately $60^{\circ}$ using the specific DLT malleable stylet (Mallinckrodt, Mansfield, MA, USA) through the bronchial lumen (Figure 1A). After we obtain the glottic view with the GVL, our first goal is to advance the tube until the tip of the bronchial lumen reaches the vocal cords, allowing the tube to engage into the glottis (Figure 1B). Next, we gently remove the stylet, which increases both flexibility and maneuvrability to the DLT and then we perform the initial $180^{\circ}$ rotation. This manoeuvre aligns the axis of the bronchial lumen with the patient's tracheal axis, facilitating the insertion of the bronchial cuff past the vocal cords (Figure 1C). Once this is accomplished, we then use the tracheal tip as the reference mark to align the tube with the left main bronchus, which usually requires an additional $90^{\circ}$ clockwise rotation. Finally, we advance the DLT until the proper depth of insertion is reached.

Contrary to the conventional method of inserting the DLT where only its alignment with the main bronchus is considered, when using the GVL, the absent line-of-sight must be taken into account during the procedure. Therefore, we suggest aligning the DLT with the trachea firstnot with the bronchus - to guarantee the insertion of the bronchial cuff into the trachea. While this is accomplished with the first rotation, the second rotation helps to align the bronchial tip with the axis of the bronchus and to move the tip away from the posterior wall of the trachea while advancing the tube until final depth.

So far, we have successfully used this DLT sequential rotation in a dozen patients without complications. The GVL was used in two patients after a failed intubation with the conventional laryngoscope, and it was used in the remaining patients as a primary airway device after suspecting a potentially difficult direct laryngoscopy. All the intubations were accomplished on first attempt. 

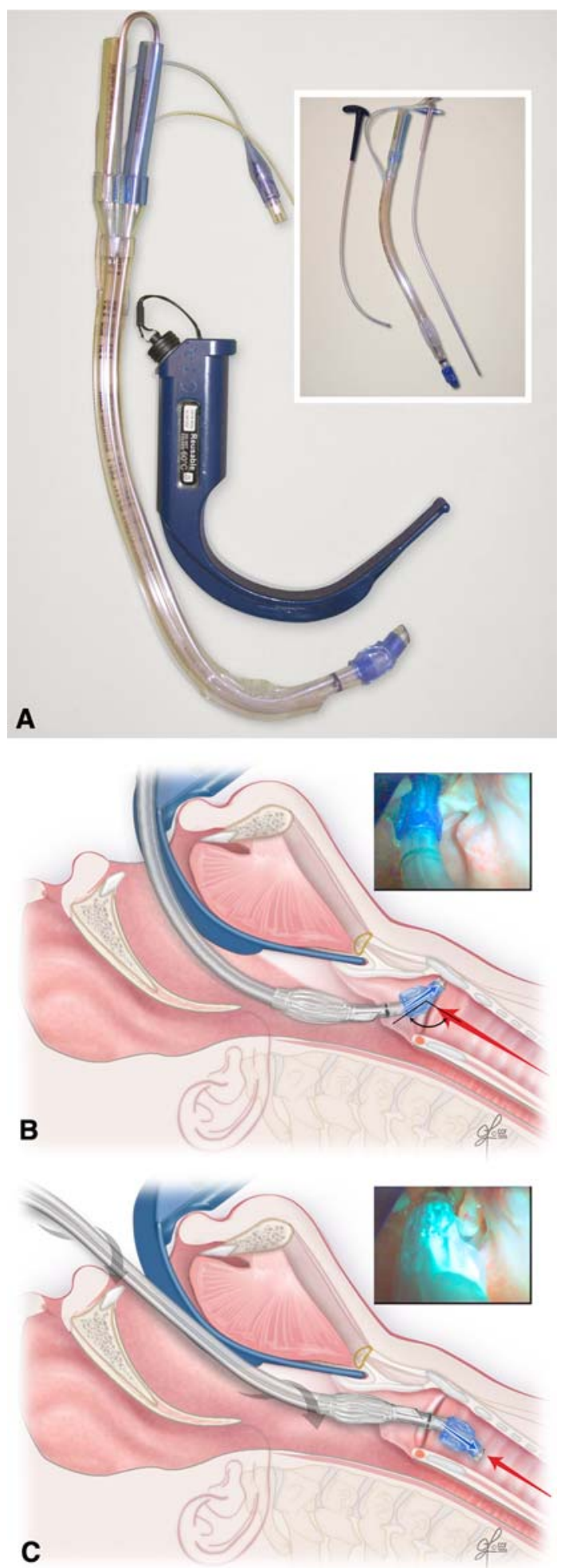

4Fig. 1 A Picture of the pre-curved tube using the specific DLT stylet. The GlideRite ${ }^{\mathrm{TM}}$ rigid stylet (Verathon, Bothell, WA, USA) may not provide the length or the flexibility required to bend the distal end of the DLT (inset). DLT = double-lumen endotracheal tube. B Illustration depicting the DLT engaging in the glottis. The tip is directed anteriorly as it reaches the vocal cords, resulting in the axis of the bronchial lumen being almost perpendicular to the axis of the trachea (arrows and inset). Aiming to align both axes will facilitate the advancement of the bronchial lumen into the trachea. DLT $=$ doublelumen endotracheal tube. C After the stylet is removed, a $180^{\circ}$ rotation of the DLT aligns the axis of its bronchial lumen with the patient's tracheal axis, facilitating its insertion (straight arrows and inset). DLT $=$ double-lumen endotracheal tube

This technique complements the traditional method of advancing the DLT, because it takes into consideration both the advantages and shortcomings of the GVL. With the expanded use of videolaryngoscopy in our clinical practice, we must adapt conventional procedures to the new technologies.

Acknowledgement We sincerely thank Mr. Jeffrey Loerch, BA, CMI for his excellent work in preparing the related medical illustrations.

Source of financial support Provided solely from departmental sources.

Conflicts of interest None declared.

\section{References}

1. Chen A, Lai HY, Lin PC, Chen TY, Shyr MH. GlideScope-assisted double-lumen endobronchial tube placement in a patient with an unanticipated difficult airway. J Cardiothorac Vasc Anesth 2008; 22: $170-2$.

2. Hernandez AA, Wong $D H$. Using a Glidescope for intubation with a double lumen endotracheal tube. Can J Anesth 2005; 52: 658-9.

3. Slinger PD, Campos JH. Anesthesia for thoracic surgery. In: Miller RD (Ed.). Miller's Anesthesia, 7th ed., vol. 2. New York: Churchill Livingstone; 2009: 1833-8.

4. Rai MR, Dering A, Verghese $C$. The Glidescope system: a clinical assessment of performance. Anaesthesia 2005; 60: 60-4.

5. Hirabayashi $Y$. The StyletScope facilitates tracheal intubation with the GlideScope. Can J Anesth 2006; 53: 1263-4. 